\title{
Test Beam Results of a Low-Pressure Micro-Strip Gas Chamber with a Secondary-Electron Emitter
}

\author{
S. Kwan, D.F. Anderson and J. Zimprerman \\ Fermi National Accelerator Laboratory \\ P.O. Box 500, Batavia, Illinois 60510 \\ C. Sbarra \\ INFN.Pisa, Via Livornese 582 \\ I-56010 S. Piero a Grado, Pisa, Italy
}

October 1994

Presented at the 1994 IEEE Nuclear Science Symposium, Norfolk, Virginia, October 30-November 5, 1994

\begin{abstract}
M. Salomon
TRIUMF

4004 Wesbrook Mall, Vancover, B.C.

Canada V6T2A3
\end{abstract}

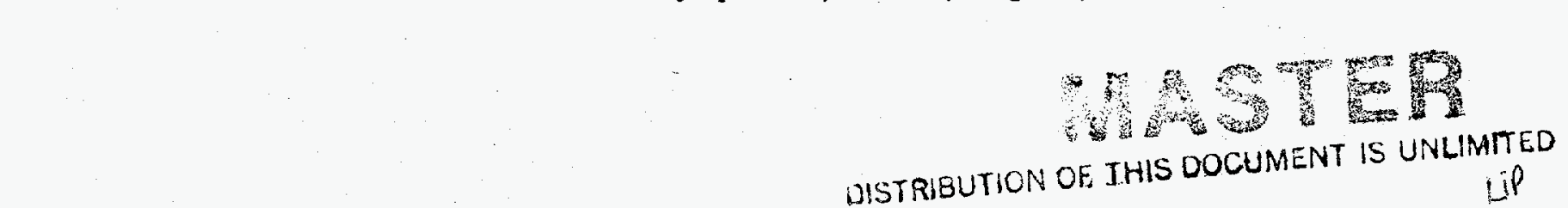




\section{Disclaimer}

This report was prepared as an account of work sponsored by an agency of the United States Government. Neither the United States Government nor any agency thereof, nor any of their employees, makes any warranty, express or implied, or assumes any legal liability or responsibility for the accuracy, completeness, or usefulness of any information, apparatus, product, or process disclosed, or represents that its use would not infringe privately owned rights. Reference herein to any specific commercial product, process, or service by trade name, trademark, manufacturer, or otherwise, does not necessarily constitute or imply its endorsement, recommendation, or favoring by the United States Government or any agency thereof. The views and opinions of authors expressed herein do not necessarily state or reflect those of the United States Government or any agency thereof. 


\section{DISCLAIMER}

Portions of this document may be illegible in electronic image products. Images are produced from the best available original document. 


\title{
Test Beam Results of a Low-Pressure Micro-Strip Gas Chamber with a Secondary-Electron Emitter
}

\author{
S. Kwan, D.F. Anderson, and J. Zimmerman ${ }^{1}$ \\ Particle Detector Group \\ Fermi National Accelerator Laboratory \\ Batavia, IL 60510 U.S.A \\ C. Sbarra \\ INFN-Pisa, Via Livornese 582 \\ I-56010 S. Piero a Grado, Pisa, Italy \\ M. Salomon \\ TRIUMF \\ 4004 Wesbrook Mall \\ Vancouver, B.C., Canada V6T 2A3
}

\begin{abstract}
We present recent results. from a beam test, on the angular dependence of the efficiency and the distribution of the signals on the anode strips of a low-pressure microstrip gas chamber with a thick CsI layer as a secondary-electron emitter. New results of CVD diamond films as secondaryelectron emitters are discussed.
\end{abstract}

\section{INTRODUCTION}

Micro-strip gas chambers, MSGC, have been shown to have a position resolution as good as to $30 \mu \mathrm{m}$ for particles of normal incidence and are able to operate at rates of up to $10^{6}$ $\mathrm{s}^{-1} \mathrm{~mm}^{-2}[1]$. Thus, they have received a great deal of attention for high energy physics in applications where tracking at high rates and over large areas is needed. One shortcoming that has hampered their application is that the single-strip efficiency, position resolution, and timing resolution degrade with increasing angle of the incident particle [2].

To address these problems we have proposed and demonstrated a low-pressure (10-20 Torr) MSGC operated with secondary-electron, SE, emission as the source of the initial ionization[3]. With this approach, we were able to increase the gas gain by more than an order of magnitude, improve the timing resolution to better than $0.9 \mathrm{~ns}$, and achieve a reduced sensitivity to discharges. The positive-ion collection time was also reduced to less than $300 \mathrm{~ns}$, yielding a shaped, anode signal as short as $10 \mathrm{~ns}$. One anticipated advantage of using $\mathrm{SE}$ emission as the source of initial ionization in a low-pressure MSGC is that the efficiency should not be dependent on the angle of the incident particle.

1 Work supported by the U.S. Department of Energy under contract No. DE-AC02-76CH03000.
The key to making the low-pressure MSGC a viable technique for high-energy physics is the discovery of an efficient and stable SE emitter that can be operated in a nonvacuum environment. To date the best emitter has been porous CsI with efficiencies of $20 \%$ or better. For non-porous CsI the efficiency is only $2-3 \%[4]$. The reader is directed to the work on SE emission by the Weizmann group (ref. [4-7]).

Here we will present the results of the first beam test of a low-pressure MSGC. We will also discuss our search for a high-efficiency SE emitter.

\section{BEAM TEST}

Measurements were made with a low-pressure MSGC in the M13 beam line at TRIUMF. The low-intensity beam consisted primarily of pions and electrons with a momentum of $100 \mathrm{MeV} / \mathrm{c}$. The trigger selected particles in a $5 \times 5 \mathrm{~mm}^{2}$ area on the face of the detector. There was not particle tracking available, so we were not able to measure the position resolution of the device. We measured the efficiency, and studied the distribution of the signals on the anodes as a function of angle.

\section{A. MSGC Configuration}

A schematic of the MSGC used for the beam test is shown in fig. 1. The details of the chamber are described in greater detail elsewhere [3]. The anode and cathode traces are $20 \mu \mathrm{m}$ and $90 \mu \mathrm{m}$ wide, respectively, with a $390 \mu \mathrm{m}$ pitch and deposited on borosilicate glass. The surface of the glass plate, with electrodes, is covered with a $50 \mathrm{~nm}$ layer of $\mathrm{Ni} / \mathrm{NiO}$ (50\%/50\%) to produce a highly resistive, ohmic surface[8]. The SE emitter was $10 \mu \mathrm{m}$ of CsI vacuum deposited on a 300 $\mu \mathrm{m} \mathrm{Si}$ substrate. The MSGC was operated with a $1.9 \mathrm{~mm}$ emitter-to-microstrip gap and a 20 Torr filling of isobutane. Cathode and emitter voltages were $-185 \mathrm{~V}$ and $-705 \mathrm{~V}$, respectively, with the signals taken from the 24 anodes at 


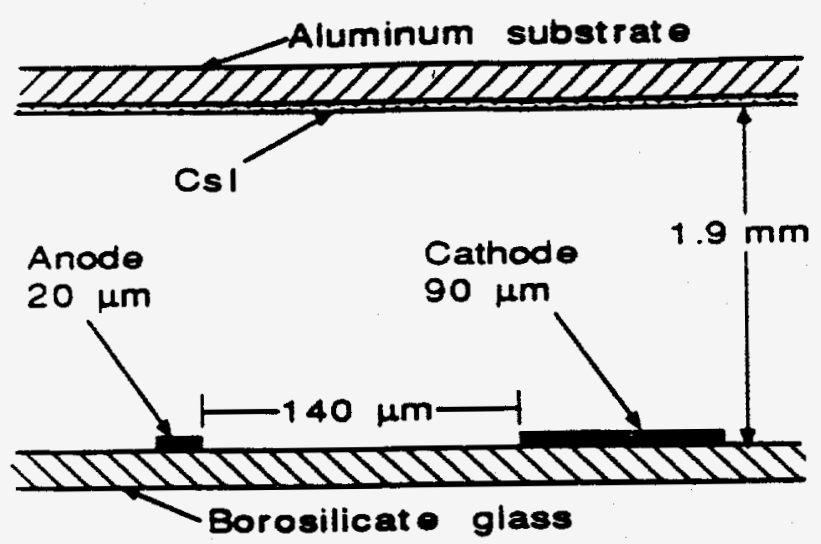

Fig. 1 Schematic of the MSGC.

respectively, with the signals taken from the 24 anodes at ground potential. Anode strips were individually read out via the QPA02 fast preamplifier [9] into a LeCroy 2249 ADC, with the trigger signal initiating the readout. The whole detector was sitting on a rotor assembly which could be controlled remotely to turn the MSGC with respect to the beam direction. In this way, the angular dependence of the chamber could be suidied.

\section{B. Test-Beam Results}

The efficiency of the MSGC was measured at angles of $0^{\circ}, 15^{\circ}$, and $30^{\circ}$ and for various pulse height cuts above the pedestal. For the analysis, we first calculated the pedestals and $\mathrm{rms}$ noise values for each anode strip. For each event, the pedestals were subtracted and for each strip, the resulting pulse height was divided by its $\mathrm{ms}$ noise value to determine the number of standard deviations, $\sigma$, above the pedestal. Beam particle hits were searched for by looking for the strip with the maximum pulse height. We could then calculate the efficiency of our chamber as a function of the number of $\sigma$ over the pedestal. The efficiency as a function of the number of sigma over the pedestal, and for the three angles, is shown in fig. 2 . For cuts below $2 \sigma$ a strong increase in efficiency was

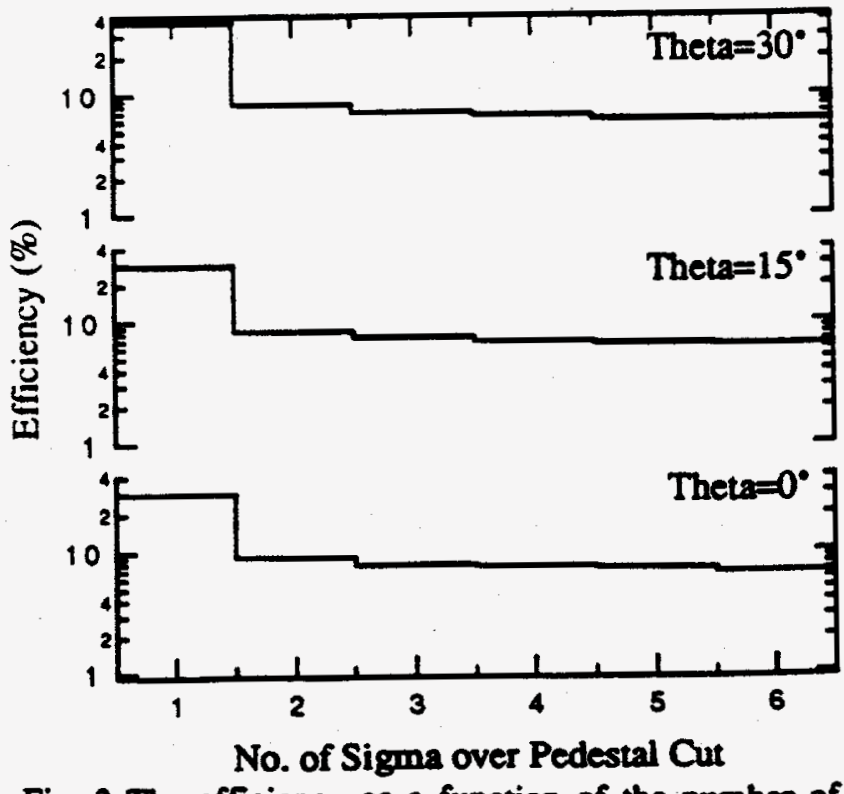

Fig. 2 The efficiency as a function of the number of sigma over the pedestal, measured at $0^{\circ}, 15^{\circ}$, and $30^{\circ}$.

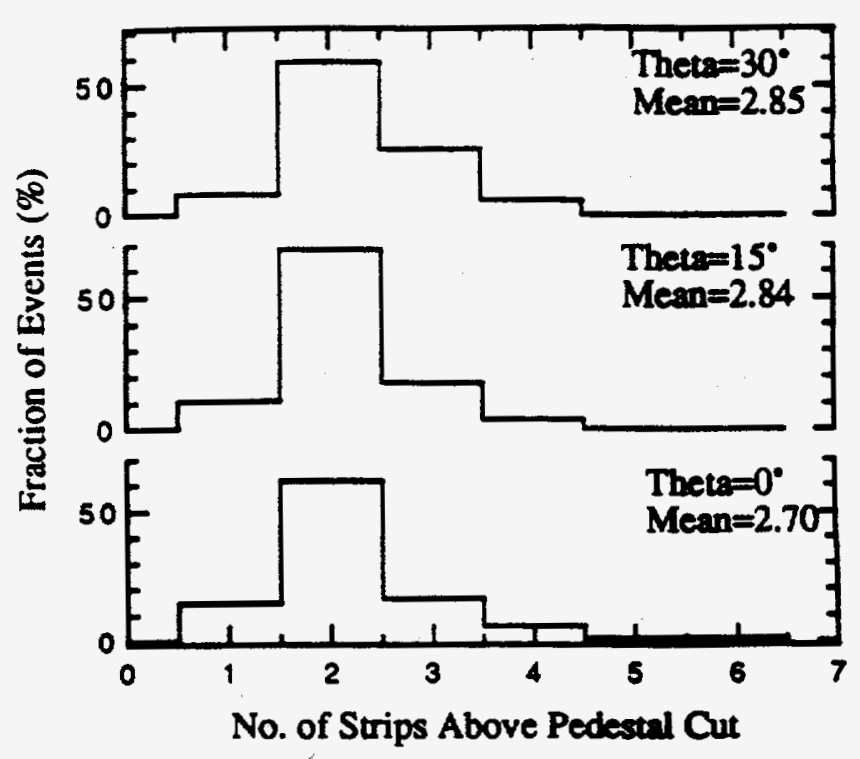

Fig. 3 Fraction of the events as a function of the number of anode strip with signals above the pedestal cut, measured at $0^{\circ}, 15^{\circ}$, and $30^{\circ}$.

observed due to noisy strips. For signals $20 \mathrm{mms}$ or greater above the pedestal, the efficiencies were $8.9 \%, 8.3 \%$, and $8.3 \%$ for $0^{\circ}, 15^{\circ}$, and $30^{\circ}$, respectively. The differences in these measurements are within the anticipated error bars of $0.5 \%$. After the test the SE emituer was tested in the lab and found to have an efficiency of $17 \%$ [10]. The discrepancy is believed to be due to the higher sensitivity of the amplifier used in the laboratory and the fact that the signal was developed on a single electrode, and not over several (see below) as in the MSGC. These measurements do show that the efficiency of the device is not angle dependent.

One feature of the low-pressure MSGC is that the charge amplification develops predominately across the gap rather than near the anode as in a MSGC operated at 1 Atm, with most of the positive ions returning to the SE emituer rather than to the adjacent cathode strips. Thus the signal is seen on more than one anode strip and potentially would allow a center-of-mass readout to yield a position resolution better than the pitch of the electrodes. Fig. 3 shows the distribution of the number of anodes strips in the cluster. The mean number of strips in the cluster were $2.70,2.84$, and 2.85 for $0^{\circ}$, $15^{\circ}$, and $30^{\circ}$, respectively. For this analysis we required that at least one strip have a signal $25 \sigma$ and the other strips counted had to have signals of $\geq 2 \sigma$. To avoid the influence of edge effects, only the central 16 strips were used in this analysis. The rms spread in the data was about 0.8 channel. Fig. 4 shows the fraction of the total cluster pulse height that is on the strip with the largest signal. The same selection of data was used as for fig. 3. For all three angles about $20 \% \pm 13 \%$ of the signal was seen in a single channel. Whether one is able to improve the position resolution with this sharing of signals will have to wait until future measurements with particle tracking. 


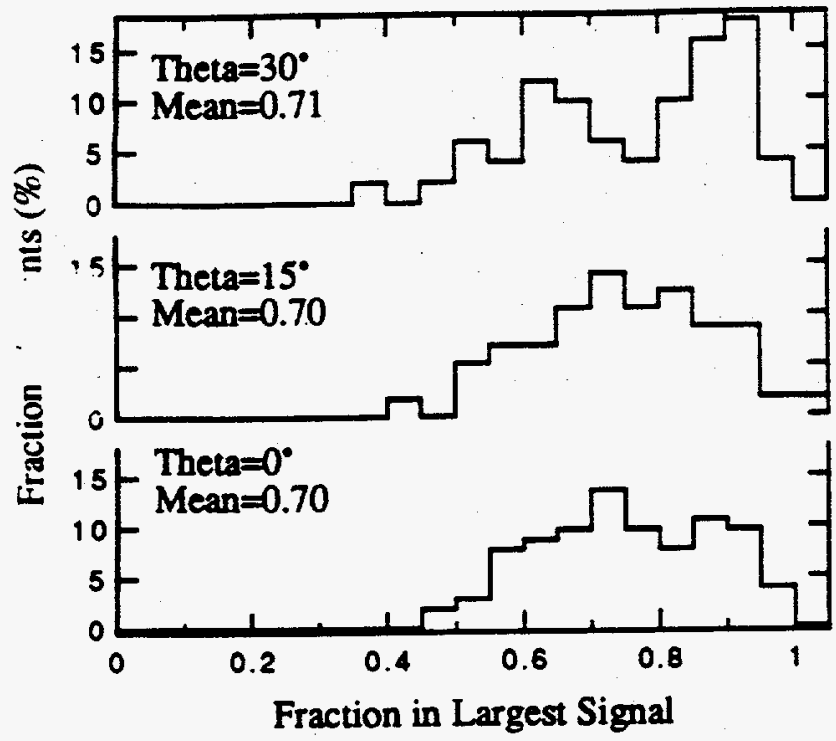

Fig. 4 Fraction of events as a function of the fraction of the total signal on the anode strip with the largest signal, measured at $0^{\circ}, 15^{\circ}$, and $30^{\circ}$.

Table I: Approximate efficiencies for minimum-ionizing particles for various emitter thicknesses

\begin{tabular}{|c|c|c|}
\cline { 2 - 3 } \multicolumn{1}{c|}{} & \multicolumn{2}{c|}{ Efriciency (\%) } \\
\hline Thicknes (A) & CSI & KCI \\
\hline 100 & 6 & 5 \\
\hline 250 & 9 & 7 \\
\hline 500 & 14 & 9 \\
\hline 1000 & 16 & 9 \\
\hline $10 \mu$ m-porous & 45 & $30 \dagger$ \\
\hline
\end{tabular}

$\dagger$ Degrades rapidly with time

\section{SEARCH FOR AN IMPROVED SE EMITTER}

As stated above, the key to making the low-pressure MSGC a viable technique is the discovery of an efficient and stable, SE emitter that can be operated in a non-vacuum environment. The best SE emitters have been the alkali halides, with CsI giving the highest yield. Using a technique described elsewhere [10] we have measured the efficiency to minimum-ionizing particles of $\mathrm{CsI}$ and $\mathrm{KCl}$ coated surfaces. Approximate efficiencies for minimum-ionizing particles for various $\mathrm{CsI}$ - and $\mathrm{KCl}$-emitter thicknesses are summarized in Table I. Our efficiencies are higher than found by others implying that our depositions are more porous, which increased the efficiency. Our samples that are labeled " $10 \mu \mathrm{m}$ porous" were deposited in 5 to 7 Tarr of argon gas rather than in a vacuum. This results in a thick, porous, low-density CsI surface, which is the best SE emituer. This still has an efficiency of only $45 \%$. Previous measurements made at Saclay in vacuum reported a stable efficiency of $62 \%$ of this type of low-density CsI emitter[11]. Thus, one can obtain an

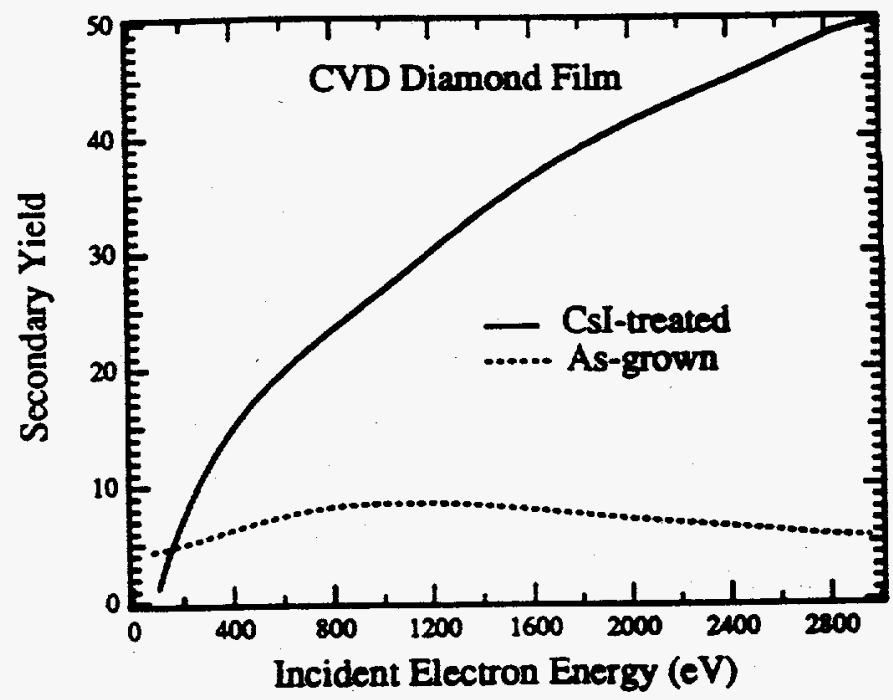

Fig. 5 Secondary yield as a function of incident electron energy for a CVD diamond film as grown and when coated with $100 \AA$ of $C s i[18]$ (see text).

efficiency for porous CsI working in a gaseous environment not too different from that obtained in vacuum. Thick, porous $\mathrm{KCl}$ also has a fairly good efficiency but degraded from $30 \%$ to only $7 \%$ in less than 30 minutes of study. We believe that this is due to charging of the surface.

In a search for a better SE emitter we have studied chemical vapor deposited, CVD, diamond films. These films are of interest because one can drift electrons in them [12-14] and the surface (in vacuum at least) has been shown to have a negative electron affinity when properly treated [15-17]. CVD diamond films have been studied both as-grown and coated with a thin coating (100A-1000A) of CsI. Measurements were made in a vacuum with the diamond surface bombarded by electrons in the $\mathrm{keV}$ energy range. This technique gives a much higher yield than from a minimum-ionizing particle. It was found that the electron beam dissociated the CsI, leaving a monolayer of Cs bonded to the diamond surface. Fig. 5 shows the secondary yield ( emitted electron / incident electron) as a function of incident electron energy for a bare and CsI-treated CVD diamond film [18]. The response of the bare diamond film is what is typically seen in such measurements: the yield increases with energy to a maximum and then decreases as the electrons become more penetrating. In the case of the CsItreated diamond there is no maximum, implying that the electrons are drifting in the diamond and then collected from the surface. (See ref. [10] for greater detail) If this can be made to work in a gas environment, we may have the SE emitter we are looking for.

\section{DISCUSSION}

A low-pressure MSGC operated with SE emission as the source of primary ionization offers high gains and a fast signal with excellent timing resolution, and reduced sensitivity to 
discharge. Although we were not able to measure the position resolution, we were able to determine that the efficiency and the details of the signal distribution did not change with increasing angle. This is an expected and encouraging result. Since the efficiency does not depend on the gas that we used, in principle, one can have a much wider range of choices of gases. The sharing of the signal on several anodes offers the hope of using center-of-mass readout techniques to obtain a position resolution better than the anode pitch, reducing the number of amplifiers (anodes) needed for a given position resolution.

The success of the low-pressure MSGC depends on an efficient and stable SE emitter. CVD diamond films appear to be very promising. We have demonsurated that, in a vacuum, one can drift electrons in the bulk and extract them from the surface. If this technique can be successfully applied to a gas environment, with the returning flux of positive ions, a SE emitter that produces as many as 36 electrons $/ \mu \mathrm{m}$ for a minimum-ionizing particle may be possible[14]. Such an emitter, coupled to a low-pressure chamber, could yield a viable MSGC that is insensitive to the angle of the incident particles, or a time-of-flight detector with a timing resolution in the 50-100 ps range.

\section{REFERENCES}

[1] RD-28. Collaboration, Development of the Micro-Strip Gas Chamber for Radiation Detection and Tracking at High Rates, CERN/DRDC193-34, 1993.
[2] F. D. v. d. Berg, et al., Nucl. Instr. and Meth A349 (1994) 438. 446.

[3] D. F. Anderson, S. Kwan, and M. Salomon, Nucl. Instr. and Meth. A346 (1994) 102.

[4] R. Chechik, et al., Nucl. Insrtr. and Meth. A342 (1994) 458.

[5] A. Akkerman et al., J. Appl. Phys. 72(11) (1992) 5429.

[6] A. Buzulutskov, A. Breskin, and R. Chechik, Field Enhancement of the Photoelectric and Secondary Electron Emission from CsL. WIS-94/11/Feb.-PH, 1994.

[7] A. Gibrekhterman, et al., The Spatial Characteristics of Electron-and Photon-Induced Secondary Electron Cascades in CsL, WIS-93/120/Dec.-PH, 1993.

[8] M. Salomon, et al., IEEE Trans. on Nucl. Sci. Conf. Record (1993) 418.

[9] T. Zimmerman, IEEE Trans. Nucl. Sci. NS-37 (1990) 439.

[10] D. F. Anderson, S. Kwan, and C. Sbarr, Low-Preasure MSCC and Search for a High-Efficiency Secondary-Electron Emitter, Intemational Conference on Advanced Technology and Particle Physics Villa Olmo, Como (Italy), 1994),

[11] C. Chienelli, et al., Nucl. Instr. and Methods A273 (1988) 245.

[12] L. S. Pan, et al. Diamond and Related Materials 2 (1993) 820.

[13] L. S. Pan, et al., J. Appl. Phys. 73(6) (1993) 1.

[14] M. Franklin, et al., Nucl. Instr. and Meth. A315 (1992) 39.

[15] B. B. Pate, et al., J. Vac. Sci. Technol. 21(2) (1982) 364.

[16] B. B. Pate, et al., Physica 117B \& 118B (1983) 783.

[17] J. v. d. Weide, and R. J. Nemanich, J. Vac. Sci. Tectnol. B10 (2) (1992) 1940.

[18] G. T. Mearini, et al., Stable Secondary Electron Emission from CVD Diamond Films Coated with Alkali-Halides, NASA LeRC Cont. no. 100, 1994. 